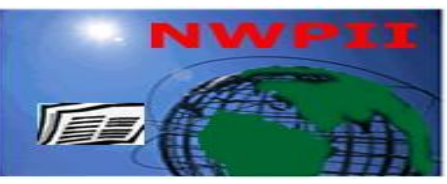

American Journal of Biomedical Sciences

ISSN: 1937-9080

nwpii.com/ajbms

\title{
Prediction of Patient's Individual Blood Glucose Levels from Home Monitored Readings of Type I Diabetics
}

\author{
A.Karim Jabali \\ Health Information Management and Technology Department, College of Applied Medical Sciences, University of \\ Dammam, Dammam, KSA \\ "Corresponding author: \\ A.Karim Jabali, Associate Professor \\ Health Information Management and Technology Department \\ College of Applied Medical Sciences \\ University of Dammam \\ Dammam 31441 \\ P.O.Box (1982) \\ Eastern Province, KSA \\ Email: aajabali@ud.edu.sa
}

Received: 4 April 2013; | Revised:13 July 2013; | Accepted: 26 July 2013

\begin{abstract}
In this paper, a comparison of two different approaches that can be used in developing time series mathematical models (MM) of diabetes mellitus was carried out. The trade-off should be considered between the complexity of the model and its accuracy to predict future glucose concentration. This work is a continuation of the author's work and results obtained previously and showed the potential and superiority of using autoregressive with exogenous terms (ARX) model in describing the dynamics of diabetes. Moreover, it is shown that despite the models are of general form but they are different depending on individuals' regimen of diabetes management. The last fact was demonstrated by using six diabetic patients' records, with rich information about their life style and treatment program, to derive models. In addition to that an answer is given to two main questions: how many future samples of glucose levels can be predicted with acceptable accuracy and what is the acceptable order of the model -complexity-, if the prediction horizon is specified. Both types of models were developed, tested and compared. This work emphasizes the fact that diabetes management plan should be formulated as an individualized therapeutic to achieve the desired level of diabetes control. This can be of help in improving the metabolic control of type-1diabetes patients by implementing these characteristics and models in both computerized controlled decision support system and simulation systems for educating and training of healthcare professional staff. Additionally, these MM of glucose-insulin interaction are expected to aid in reaching a generalized model.
\end{abstract}

Keywords: diabetes, time series, modeling, prediction. 


\section{Introduction}

Diabetes mellitus (DM) is a term applied to a number of conditions or symptoms that, in untreated state, are known by hyperglycemia. Hyperglycemia is characterized by the inability of the pancreas to control blood glucose concentration. In other words, it is a disorder of metabolism of carbohydrate, fat and protein associated with a relative or absolute insufficiency of insulin secretion and with various degrees of insulin resistance. Insulin is a hormone that is produced in the beta cells of the pancreatic islets of Langerhans. Its role is twofold; firstly to enhance the entry of glucose into the liver, muscle and adipose tissue, and secondly to promote storage of energy substrate in the form of glycogen, fat and protein thus resulting in a lowering of the blood glucose concentration. It is very important to keep blood glucose concentrations within a range from 60 $\mathrm{mg} / \mathrm{dL}$ to $120 \mathrm{mg} / \mathrm{dL}$, depending on when a person last ate [1]. Lower blood glucose concentrations (hypoglycemia) impair brain function, whereas higher glucose concentrations (hyperglycemia) exceed the renal glucose reabsorption threshold, which results in wasting of glucose. In addition, protracted hyperglycemia causes degenerative complications in the longterm and may cause damage to the eyes, kidneys, nerves, heart and blood vessels $[2,3]$. There are two major forms of diabetes mellitus: type 1 and type 2 DM; Type 1 diabetes mellitus (formerly called Insulin Dependent Diabetes Mellitus, IDDM, or juvenile diabetes) is characterized by destruction of the pancreatic beta cells caused by an autoimmune process, usually leading to absolute insulin deficiency. Type 2 diabetes mellitus (formerly called Non-Insulin Dependent Diabetes Mellitus, NIDDM, or adult-onset diabetes) is characterized by insulin resistance in peripheral tissue, and an insulin secrecy defect of the pancreatic beta cells. The latter is the most common form of diabetes mellitus, and is highly associated with a family history of diabetes, older age, obesity and lack of exercise.

Despite recent general improvements in diabetes care, it remains one of the leading causes of death, main cause of new blindness, kidney failure, and amputations and poses as a major risk factor for heart disease, stroke and birth defects [4-6]. In the same time and while all patients and medicine community look for a solution to diabetes problem, the main effort of physicians still focused on controlling blood glucose level which can substantially reduce the risk of developing complications and slow their progression in diabetics. The control, in its natural meaning, requires the determination of related inputs, processes, and outputs of diabetes metabolism, or in control engineering terms, the discovery of system's dynamics. This leads to build a mathematical model that reflects the understanding of interrelationships among most if not all factors that have impact on diabetes as a process.

Although all diabetic patients share a common goal to gain control of their blood sugar, it is agreed that every patient has individual treatment program that works best for him or her $[7,8]$. In addition to that this individualism is not of a constant non-changing nature but changes with time (age), growth, and other factors. Many individual patient's characteristics and factors are relevant for achieving blood sugar control. This includes, but is not restricted to, prior glucose levels records, eating patterns, physical exercise, current treatment of diabetes including insulin, oral glucose-lowering agents, glucagon and other medications, and results of glucose monitoring. An individualized patient action plan should be developed and patients should be instructed and/or taught within this action plan on how to adjust their regimen in response to results from self-monitoring of blood glucose, when a large meal is eaten, or during illness.

This work investigates the individual's life style and treatment program history impact on developing one specific mathematical model category, i.e. time series models that are a strong candidate to manage and hence improve metabolic control of glucose level. Moreover, one of the objectives of this work is to verify that different patients have different parameters but of the same time series category model.

Models are indispensable for scientific research and they are of more importance for biomedicine as it deals usually with complex 
systems. The objectives of mathematical models are to represent and predict the profile of a drug in a patient or group of patients. Consequently, mathematical models are used in attempting to mimic the real natural process by using the simplest model defined by a mathematical formula. Biologically realistic mathematical models serve as the basis for the majority of the methods used in quantitative physiologic analyses of medical data $[9,10]$. These models help to test hypotheses about the mechanisms that govern these complex systems. They help to identify contradictions or incompleteness in data testing of hypotheses, and allow prediction of system performance under either untested or practically un-testable conditions. They may also predict and supply the values of experimentally inaccessible variables. The more and the deeper the knowledge of the biomedical system, the more potential and beneficial is the use of the MM.

A wide diversity of mathematical models has been proposed to describe DM as a biomedical phenomenon. These models range from models that consist of a set of linear differential equations to models of nonlinear differential equations [11-13]. Other classes of models like time-series models [14], probabilistic models [15] and non-compartmental models [16] have also been used. Moreover, there are other research about the impact of DM on other diseases and illnesses [17]. In the last two decades, several research groups have used, and continue to use, new modeling and identification techniques that are based on artificial intelligence[18]. These include fuzzy logic[19, 20], neural networks [21$24]$ and a combination of these and other methods [25-27]. All these models, including those developed in this paper, are built around glucose metabolism and insulin kinetics. They are intended to estimate the time course of glucose concentration and are expected to be conclusive to a generalized model (secret formula) that explores the internal relationships among such factors like the insulin dose and its types, exercise, meal, and other relevant factors. Recent developments in diabetes modeling are reported in several papers [28-32].

In the specialized areas of diabetes modeling, the time series models have been the most commonly used. This is because they make use of time history of the patient's measured data, in addition to the fact that these models are of wide variety and have yielded promising results [9, 33]. In other words, the comprehensive medical history can uncover symptoms that help establishing the diagnosis of the patient's case. Moreover, the history should confirm the diagnosis, review the previous treatment, allow evaluation of the past and present degrees of glycemic control, determine the presence or absence of the chronic complications of diabetes, assist in formulating a management plan, and provide a basis for continuing care.

Patients with type 1 diabetes require more or less intensive insulin regimens to maintain optimal blood glucose control. The goal of insulin therapy is to mimic normal insulin secretion by the pancreas. Normal insulin secretion consists of basal and mealtime insulin secretion [34]. Self-monitoring of blood glucose is recommended several times daily, because many factors, such as food consumption, Exercise, illness, stress, and alcohol, can influence insulin requirements.

There are many forms of insulin used to treat diabetes. They are classified by how fast they start to work and how long their effects last. The types of insulin include: Short-Term acting Insulin (STI), Mid-Term Insulin (MTI) and LongTerm acting Insulin (LTI).

\section{Data and methods}

In order to investigate the individualism of diabetes management, a data from six type 1 home monitoring diabetics' were used. The data covers a period of about 3 months. During that time, the Present blood Glucose Level (PGL), dosages of insulin injections (STI, MTI, and LTI), qualitative indication about the amounts of food intake (Meal) and physical effort exerted (Exercise) were recorded [35]. The data reflects the general parameters that can be either measured or estimated and therefore managed by the patient himself. The glucose and insulin values are given precisely but the amount of food and extend of physical exercise were estimated high, medium or low. 
The goal of this study is to use the real data collected from patients to develop empirical dynamic autoregressive exogenous input (ARX) time-series models of these diabetics. After that, obtained models to be analyzed and compared from the point of view of their efficiency in addition to their mathematical properties. The efficiency of the developed models is tested by its ability to predict the future glucose level readings for specified parameters values as well as to perform parameter estimation in which model output are fit to the clinical data. The input to the system in all developed models consists from PGL, STI, MTI, LTI, Meal, and Exercise, while the output is the next value of PGL or it can be called Next Glucose Level (NGL). In this study the data is divided into two sets: one called estimation data used to estimate the formula coefficients, and the other called validation data used to find how fit is the given formula in predicting the output. Two different multivariate time-series models of glucose/insulin dynamics are developed and compared for all patients in the study.

The Auto-Regressive with Exogenous input (ARX) model is used in this study. The use of this type of models is justified because of its relative simplicity and is power in performing sufficiently accurate predictions [36].Two aspects of these models were investigated. They are the variability of the model's order and the model's performance with constant order. This was done by using, firstly ARX model with fixed order to answer the question: how perfect can the model predict fixed number of NGL values of glucose levels. Secondly, the order of the used model was assumed to be changeable factor but the number of NGL values that can be predicted was fixed. In the last case, five and ten NGL values of blood glucose concentration were tested. All these simulations were carried out using MATLAB software package.

\section{ARX modeling and validation}

A common question that justifies the use of ARX models is whether present and future glucose values can be predicted from recent blood glucose history. The fact that there is significant statistical inter-dependence among the individual successive glycemic measurements motivates the use of patient's record to find a model that at least forecasts the near future values of glucose. The latter might be helpful for both the patient and physician in dealing with the complications of the disease. A multivariable ARX model is given by

$$
A(q) y(t)=B(q) u(t-n k)+e(t)(1)
$$

where $\mathrm{u}(\mathrm{t}), \mathrm{y}(\mathrm{t})$, and $\mathrm{e}(\mathrm{t})$ are input, output and random disturbance vectors respectively, $n k$ is the number of delays from input to output and $A(q), \mathrm{B}(\mathrm{q})$ are the corresponding polynomials , to be determined (estimated), in the delay operator $q^{-1}$.But the model, after that, is to be validated. One principal method to accomplish the task of validation is to test prediction properties of the model i.e. the response of the system to the validation data set which is usually different from that used for parameter estimation. Hence, prediction is necessary to evaluate how well the proposed model is capable of predicting future values of the observed data. This prediction method depends on the model structure[37]. The performance of the model is to be assessed using the following measures

\section{1-Mean Square Error (MSE)}

$$
\mathrm{MSE}=\sum{\text { ( } \left.\text { Error. }^{\wedge} 2\right)}^{\wedge} \text { / length(ANGL) }
$$

2-Mean Absolute Error (MAE)

$$
\mathrm{MAE}=\sum \mid \text { Error } \mid / \text { length }(\mathrm{ANGL})
$$

\section{3-Percentage Relative Error}

$$
\text { PRE }=\mid((\text { PNGL }- \text { ANGL. } / \text { ANGL }) \mid * 100
$$

where PNGL and ANGL are Predicted and Actual Next Glucose Level and the Error is defined as

$$
\text { Error }=\mathrm{ANGL}-\mathrm{PNGL}
$$




\section{Results}

Figure (1) shows the actual data set compared with the predicted values using ARX model with a length up to 60 future values. The figure shows that the ability to predict upcoming values is possible but the choice is between the model order and length of accurately predicted values of glucose concentration.

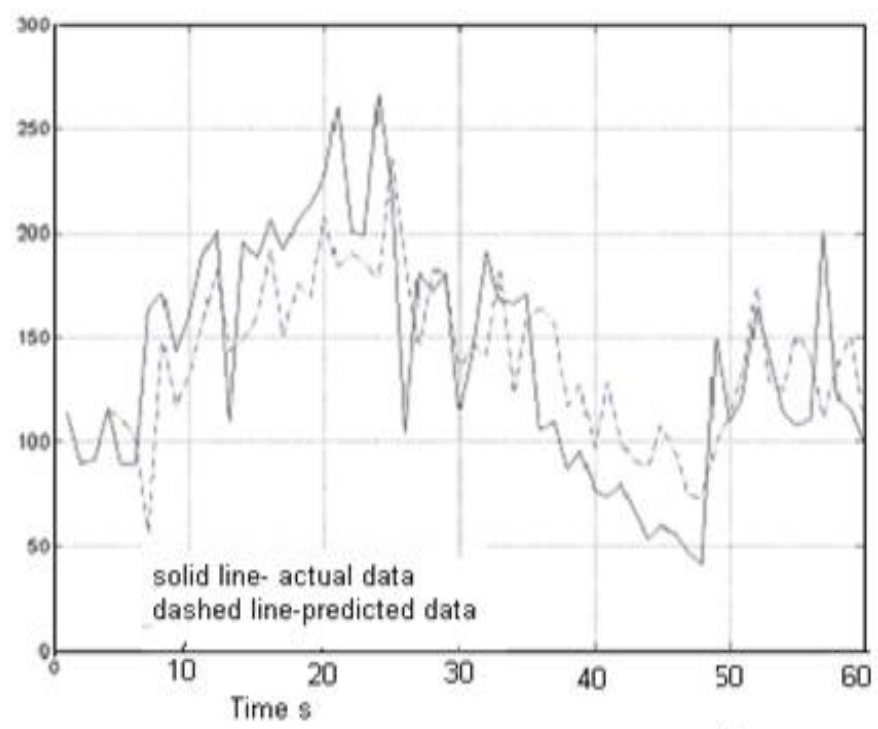

Figure 1. Actual and Predicted Data for ARX Model

\subsection{Fixed Order ARX}

Here the modeling was carried out for 6 patients with the same model order, third order, and then the performance of all cases was tested to explore the individual differences between patients. The ability of the models to predict 10 future values of glucose concentration was the criterion; Table (1) shows the quantitative measures of the models for patients P1-P6, where figure (2) shows the first ten actual and predicted data for ARX model.

It is clear that MSE gives values that are different not only in value but also in the order i.e. the last patient P6 and P3 have a value of hundreds while other four patients have a value of thousands. The individual character of patients is reflected clearly. Moreover other measures of performance are consistent with this conclusion.
TABLE 1. Ten steps arx models performance

\begin{tabular}{|l|l|l|l|}
\hline \multicolumn{4}{|c|}{ 10 Steps ARX Models } \\
\hline Performance & MSE & MAE & PRE \\
\hline P1 & $2.6196 \times 10^{3}$ & 33.8207 & 2.3973 \\
\hline P2 & $2.4463 \times 10^{3}$ & 33.8676 & 0.3984 \\
\hline P3 & 846.0165 & 20.5047 & 0.8504 \\
\hline P4 & $4.6213 \times 10^{4}$ & 172.0476 & 22.7075 \\
\hline P5 & $3.6833 \times 10^{3}$ & 43.4477 & 0.2999 \\
\hline P6 & 340.6306 & 12.8987 & 0.1053 \\
\hline
\end{tabular}

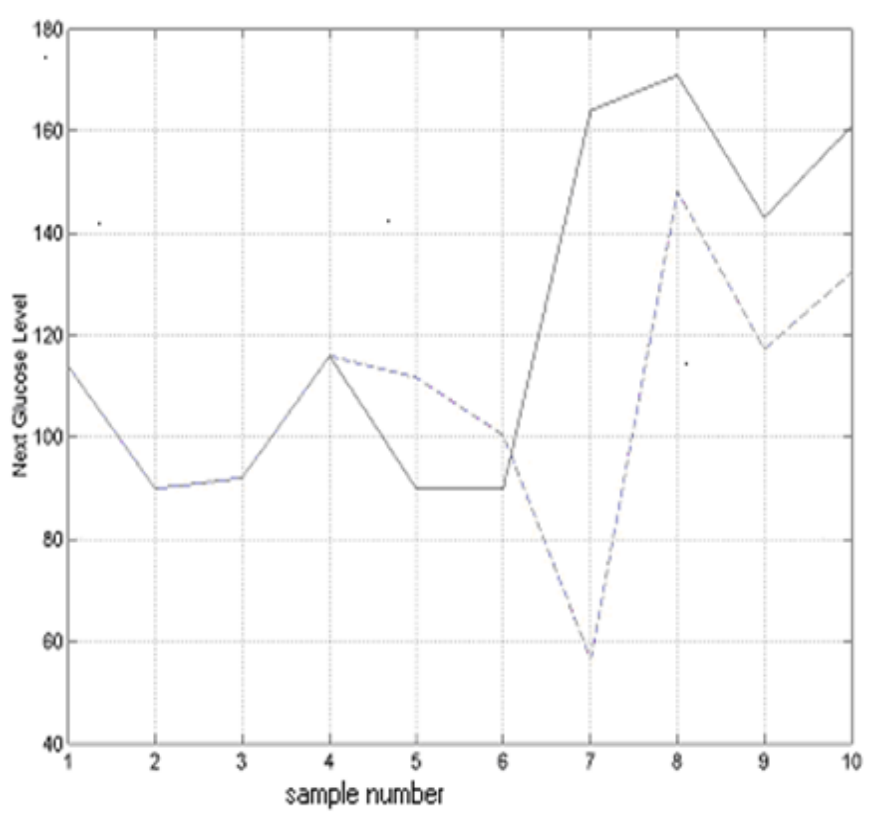

Figure 2. First 10 actual data and predicted data for the ARX model. 
Table 2: Order of ARX models with fixed prediction horizon.

\begin{tabular}{|l|l|l|l|}
\hline patient & $\begin{array}{l}\text { Order of } \\
\text { polynomial } \\
\boldsymbol{A}\end{array}$ & $\begin{array}{l}\text { Order of } \\
\text { polynomial } \\
\boldsymbol{B}\end{array}$ & $\begin{array}{l}\text { Number of } \\
\text { delays } \\
\boldsymbol{n} \boldsymbol{k}\end{array}$ \\
\hline P1 & 1 & 9 & 1 \\
\hline P2 & 2 & 9 & 2 \\
\hline P3 & 1 & 9 & 1 \\
\hline P4 & 1 & 9 & 1 \\
\hline P5 & 2 & 9 & 4 \\
\hline P6 & 8 & 9 & 9 \\
\hline
\end{tabular}

\subsection{Variable Order ARX}

The_second group of models with variable order is intended to show that changing the order can be helpful in achieving exact prediction of some number of future values. The models were developed with the order that gives precise prediction of five future values of glucose concentration. Table 2 shows that these orders are different and the individual aspects are obvious. The order of the input polynomial, A, for the first patient, for example, is one, while the last patient's order is eighth and for other polynomials the difference between patients is very clear. After that, the ability of the obtained models was evaluated for the case of 10 future values of the output and summarized in Table 3 which indicates the decreased values of MSE and other performance criteria for the same patient. Moreover, for one patient (P6), the model's ability to predict 10 values is excellent, i.e. with very small value of MSE and other performance indices.

\section{Discussion and Conclusions}

The above results bring out two options for developing the appropriate algorithm of therapy.
The first is to use the ARX model with fixed order to predict the future course of glucose concentration but the horizon of accurate future values is from three to five values or near half a day time values. Here it's worth to emphasizing on the fact that the model used is of low order, third order, which has low computational load to the decision support system while other studies are using more higher order models or don't consider this factor [38, 39]. The next choice offers longer horizon starting from 5 and may be 10 or more if necessary but the order of the model is increased. In both cases the availability of accurate measurements (history) of the blood glucose is essential. Treatment regimen is to be carried out individually; this reduces the patient's risk for severe hypoglycemia, and may increase benefits of glycemic management and control. People with diabetes should work with a health care provider to find the appropriate individual patient targets and how to achieve them. Elements of the medical history of particular concern in patients with diabetes include the following prior glucose levels records, eating patterns, physical Exercise history current treatment of diabetes, including medications, (insulin, , oral glucose- lowering agents, glucagon and other medications, and results of glucose monitoring.

In order to extend the done work, consideration can be given additionally to the patient's age, school or work schedule and other conditions, physical activity, eating patterns, social situation and personality, cultural factors, and presence of complications of diabetes or other medical conditions. With the goal of achieving near normoglycemia and delaying the onset and the progression of many complications, of type 1 diabetes, this future task requires precise quantitative and/or qualitative recording and/ or estimating of these factors. 
Table 3: Comparison of the five and ten steps models

\begin{tabular}{|l|l|l|l|l|l|l|}
\hline \multicolumn{2}{|l|}{} & \multicolumn{2}{l}{5 step model } & \multicolumn{2}{l|}{10 step model } \\
\cline { 2 - 7 } & MSE & MAE & PRE & MSE & MAE & PRE \\
\hline P1 & 0 & 0 & 0 & 378.57 & 8.2704 & 0.8439 \\
\hline P2 & 0 & 0 & 0 & 72.3978 & 2.5655 & 0.2171 \\
\hline P3 & 0 & 0 & 0 & 158.5636 & 5.3377 & 0.5544 \\
\hline P4 & 0 & 0 & 0 & $1.1209 \times 10^{3}$ & 12.4691 & 1.6270 \\
\hline P5 & 0 & 0 & 0 & $7.3436 \times 10^{-28}$ & $1.5503 \times 10^{-14}$ & $6.1570 \times 10^{-16}$ \\
\hline P6 & 0 & 0 & 0 & $3.6718 \mathrm{e}-029$ & $2.5838 \mathrm{e}-015$ & $2.5577 \mathrm{e}-016$ \\
\hline
\end{tabular}

\section{References}

1. Robbins, C., Pathologic Basis of Disease. 8 ed2009, USA: Elsevier,.

2. Centers for Disease Control and Prevention. [cited 2012 November,12]; Available from: 2. http://www.cdc.gov

3. Jean Venable, R., Goode, Pharm D, New Advances in the Treatment of Diabetes. 2001.

4. Diabetes in America. [cited 2010 November,20]; Available from: http://www.niddk.nih.gov,2010.

5. World Health Organization Fact Sheet $N^{\circ}$ 310, 2008, www.who.org

6. G Imperatore, J.B., TJ Thompson,, Projections of Type 1 and Type 2 Diabetes Burden in the US Population Aged $<20$ Years Through 2050 Dynamic modeling of incidence, mortality, and population, Diabetes
Care, 2012. 35(12): p. 2515-20. DOI $10.2337 / \mathrm{dc} 12-0669$.

7. GC Weir, D.N., and DE Singer, Standards of care for diabetes, Diabetes Care. 17: p. 15141522 ,

8. Brown, A.B., Individualizing insulin therapy for optimum glycemic control. Patient Care Archive. 1: p. 35-47.

9. Modeling in Biomedical Research: An Assessment of Current and Potential Approaches: Applications to Studies in Cardiovascular/Pulmonary Function and Diabetes. in Proceedings of National Institutes of Health Conference.

10. Tyler Bevan, R.C., Lee Goneau, Peter Cadieux, Hassan Razvi, Numerical Simulation of Peristaltic Urine Flow in a Stented Ureter AMERICAN JOURNAL OF 
BIOMEDICAL SCIENCES, 2012. 4(3). http://ww.nwpii.com/ajbms/papers/AJBMS

11. Candas B, R.J., An adaptive plasma glucose controller based on a nonlinear insulin/glucose model. IEEE TA BME, 1994(41): p. 116-124.

12. Thomas Briegel, V.T.A., Nonlinear State Space Model for the Blood Glucose Metabolism of Diabetic. Automatisierungstechnik, 2002. 50(5).

13. R.S.Parker, F.J.D., and N.A.Peppas, A Modelbased Algorithm for Blood Glucose Control in Type 1 Diabetic Patients. IEEE TA BME, 1999. 46(2).

14. Riccardo Bellazzi, P.M., Giuseppe De Nicolao; Bayesian Analysis of Blood Glucose Time Series from Diabetes Home Monitoring IEEE TA BME, 2000. 47(7).

15. Andreassen S, B.J., Hovorka R, Olesen K, Carson E, A probabilistic approach to glucose prediction and insulin dose adjustment: description of a metabolic model and pilot evaluation study. Computer Methods and Programs in Biomedicine, 1994. 41: p. 153-163.

16. Cobelli C, T.G., Ferrannini E, A model of glucose kinetics and their control by insulin, compartmental and noncompartmental approaches. Mathematical Biosciences, 1996. 71: p. 291-316.

17. Almarzouki, A.A., Glycated Hemoglobin "Alc" and Diabetes Complications AMERICAN JOURNAL OF BIOMEDICAL SCIENCES, $2012 . \quad 4(2)$. http://ww.nwpii.com/ajbms/papers/AJBMS

18. Kovacs, L.G., A. ; Benyo, B. ; Benyo, Z. ;Kovacs, A Soft computing control of Type 1 diabetes described at molecular levels, in SACI '09 Applied Computational Intelligence and Informatics, 2009.

19. A.Karim Jabali A. Kailani, M.A., Dynamic simulation of fuzzy controller of glucose concentration based on patient's clinical database, in Modeling and Simulation Conference MS'02; 2002.

20. Lee CS, W.M., A Fuzzy Expert System for Diabetes Decision Support Application. IEEE Transactions on Systems, Man, and Cybernetics,, Feb. 2011. Part B: Cybernetics.
21. J;P., Modeling of blood glucose levels using artificial neural networks, 1997, University of Strathclyde,Scotland.

22. V. Tresp, T.B.a.J.M., Neural Network Models for Blood Glucose Metabolism of Diabetic. IEEE TA on Neural Networks, 1999. 10(.5. ): p. 1204--1213, .

23. K.Y. Chana, S.H.L., T.S. Dillona, and H.T. Nguyenb, Diagnosis of hypoglycemic episodes using a neural network based rule discovery system. Expert Systems with Applications, February 2011,. 38, (Issue 8,): p. 9799-9808.

24. Scott M. Pappada, B.D.C., Paul M. Rosman, D.O, M.D. Raymond E. Bourey, Thomas J. Papadimos, M.D., M.D. William Olorunto, Marilyn J. Borst, M.D., Neural NetworkBased Real-Time Prediction of Glucose in Patients with Insulin-Dependent Diabetes. DIABETES TECHNOLOGY \& THERAPEUTICS,2011. 13(1). DOI: 10.1089/dia.2010.0104

25. R.J.Frank, N.D., S.P.Hunt, Time Series Prediction and Neural Networks, 1997., Department of Computer Science, University of Hertfordshire, Hatfield, : UK.

26. Duygu Çalişira, E.D., An automatic diabetes diagnosis system based on LDA-Wavelet Support Vector Machine Classifier. Expert Systems with Applications, 2011. 38(7).

27. Vahidi, O., Dynamic Modeling of Glucose Metabolism for the Assessment of Type II Diabetes Mellitus, 2013, University of British Columbia. http://hdl.handle.net/2429/43995

28. Naylor JS, H.A., Albisser AM, Evers JH, Strickland JH, Schumacher DA:, Comparison of parameterized models for computer-based estimation of diabetic patient. Glucose response. Medical Informatics,1997. 22: p. 21-34.

29. Hipszer, B., A type 1 Diabetic Model, 2001, Drexel University; .

30. Arabasy, H., LMI-Based Control of nonlinear time delay model for "type I diabetic patient, 2010, KFUPM, KSA

31. Cobelli, C.D.M., C.; Sparacino, G.; Magni, L.; De Nicolao, G.; Kovatchev, B.P., Diabetes: Models, Signals, and Control. 
Biomedical Engineering, IEEE Reviews in, 2009. .2, : p. 54-96.

32. Joseph El Youssef, C.J., W Kenneth Ward, A Review of Closed-Loop Algorithms for Glycemic Control in the Treatment of Type 1 Diabetes Algorithms, 2009. 2009(2): p. 518532. DOI:10.3390/a2010518

33. Duke, D.L., Intelligent Diabetes Assistant, A Telemedicine System for Modeling and Managing Blood Glucose,The Robotics Institute,2009, Carnegie Mellon UniversityPittsburgh, Pennsylvania

34. Singla, D., Individualizing Insulin Therapy in Type 1 Diabetes Mellitus April; 1st, 2005 ,, in pharmacy times 2005

35. Kahn;, M., Artificial Intelligence in Medicine in AIM-941994, Washington University: Washington University.

36. Harvey, A.C., Time Series Models, . 2nd ed1992: Harvester Wheatsheaf; .
37. El-Jabali, A.K., Development of Diabetes Mellitus Mathematical Models From Patient's Clinical Database. Informatica journal of computing and information technology, 2004. Volume 28 (2).

38. Gani, A.G., A.V. ; Yinghui Lu ; Ward, W.K. ;Vigersky, R.A. ; Reifman, J, Universal Glucose Models for Predicting Subcutaneous Glucose Concentration in Humans IEEE Transactions on Information Technology in Biomedicine, 2010. 14(1).

39. Elena Daskalaki, A.P., Peter Diem, and Stavroula G. Mougiakakou, Real-Time Adaptive Models for the Personalized Prediction of Glycemic Profile in Type 1 Diabetes Patients J Diabetes Sci Technol., 2012. 6(3): p. 617-633. 